\title{
Probing the proper motion of the central compact object in Puppis-A with the Chandra high resolution camera
}

\begin{abstract}
C. Y. Hui and W. Becker
Max-Planck Institut für Extraterrestrische Physik, Giessenbachstrasse 1, 85741 Garching bei München, Germany e-mail: dhui@mpe.mpg.de

Received 30 June 2006 / Accepted 8 August 2006

\section{ABSTRACT}

By making use of the sub-arcsecond angular resolution of the High Resolution Camera (HRC-I) aboard the Chandra X-ray satellite we have examined the central compact object RX J0822-4300 in the supernova remnant Puppis-A for a possible proper motion. Using data which span an epoch of 1952 days we found the position of RX J0822-4300 different by $0.574 \pm 0.184$ arcsec, implying a proper motion of $\mu=107.49 \pm 34.46 \mathrm{mas} / \mathrm{yr}$. For a distance of $2.2 \mathrm{kpc}$, this proper motion is equivalent to a recoil velocity of $1121.79 \pm$ $359.60 \mathrm{~km} \mathrm{~s}^{-1}$. The position angle is found to be $241^{\circ} \pm 24^{\circ}$. Since both the magnitude and direction of the proper motion are in agreement with the birth place of RX J0822-4300 being near to the optical expansion center of the supernova remnant, the result presented in this letter is a promising indication of a fast moving compact object in a supernova remnant. Although the positional shift inferred from the current data is significant at a $\sim 3 \sigma$ level only, one or more future HRC-I observations can obtain a much larger positional separation and further constrain the measurement.
\end{abstract}

Key words. pulsars: individual: RX J0822-4300

\section{Introduction}

The group of supernova remnant (SNRs) which are known to host a radio-quiet but $\mathrm{X}$-ray bright central compact object (CCO) is a slowly growing one. Thanks to more sensitive X-ray observatories it currently includes Cas-A (CXOU J232327.9+584842; Tananbaum 1999), RX J0852.0-4622 (CXOU J085201.4-461753; Aschenbach 1998), RX J1713.7-3946 (RX J1713.4-3949; Pfeffermann \& Aschenbach 1996), RCW 103 (1E 161348-5055; Tuohy \& Garmire 1980), Puppis-A (RX J0822-4300; Petre et al. 1982, 1996; Hui \& Becker 2006), PKS 1209-51/52 (1E 1207.4-5209; Helfand \& Becker 1984) and Kes 79 (CXOU J185238.6+004020; Seward et al. 2003; Gotthelf et al. 2005).

RX J0822-4300 was first noticed in one of the EINSTEIN HRI images of the Puppis-A supernova remnant (Petre et al. 1982). However, it appeared in this data only as a faint X-ray feature. It is not until the era of ROSAT when it became evident that RX J0822-4300 is the central compact stellar remnant which was formed in the supernova event (Petre et al. 1996).

Recently, Hui \& Becker (2006) presented results from a detailed analysis of RX J0822-4300 which made use of all XMM-Newton and Chandra data available from it by beginning of 2005. The spectral analysis of XMM-Newton data revealed that the X-ray emission from RX J0822-4300 is in agreement with being of thermal origin. A double blackbody model with the temperatures $T_{1}=(2.35-2.91) \times 10^{6} \mathrm{~K}, T_{2}=$ $(4.84-5.3) \times 10^{6} \mathrm{~K}$ and the projected blackbody emitting radii $R_{1}=(2.55-4.41) \mathrm{km}$ and $R_{2}=(600-870) \mathrm{m}$ gave the best description of the observed point source spectrum among various spectral models tested. The X-ray images taken with the Chandra HRC-I camera allowed for the first time to examine the spatial nature of RX J0822-4300 with sub-arcsecond resolution. Besides an accurate measurement of the source position, this observation constrained the point source nature of RX J0822-4300 down to $0.59 \pm 0.01 \operatorname{arcsec}(\mathrm{FWHM})$ for the first time.

Despite the effort in searching for coherent radio pulsations, RX J0822-4300 has not been detected as a radio pulsar (Kaspi et al. 1996). Similar to many other CCOs, it has no optical counterpart down to a limiting magnitude of $B \gtrsim 25.0$ and $R \gtrsim 23.6$ (Petre et al. 1996). Together with the lack of long term flux variation (Hui \& Becker 2006), all these evidences rule out many types of X-ray sources as a likely counterpart of RX J0822-4300, except a neutron star.

RX J0822-4300 is located about $\sim 6$ arcmin distant from the optical expansion center of Puppis-A, which is at RA = $08^{\mathrm{h}} 22^{\mathrm{m}} 27.45^{\mathrm{s}}$ and Dec $=-42^{\circ} 57^{\prime} 28.6^{\prime \prime}(\mathrm{J} 2000)(\mathrm{cf}$. Winkler \& Kirshner 1985; Winkler et al. 1988). The age of the supernova remnant, as estimated from the kinematics of oxygen-rich filaments is $\sim 3700$ years (Winkler et al. 1988). If these estimates are correct, RX J0822-4300 should have a proper motion of the order of $\sim 100$ mas/yr to a direction away from its proposed birth place.

In this letter we test this hypothesis by making use of archival Chandra HRC-I data spanning an epoch of somewhat more than five years. The expected positional displacement for RX J0822-4300 in this time span is of the order of $\sim 0.5$ arcsec. This is in the range of the Chandra accuracy given the possibility to correct for pointing uncertainties by using X-ray counterparts of stars with accurate position and which are serendipitously located in the field of view. 
Table 1. X-ray properties of serendipitous sources in the neighborhood of RX J0822-4300.

\begin{tabular}{lcccc}
\hline \hline Source & \multicolumn{2}{c}{ X-ray position } & Positional error & \\
& RA $(\mathrm{J} 2000)$ & Dec $(\mathrm{J} 2000)$ & $\begin{array}{c}\text { Net count rate } \\
\text { cts/s }\end{array}$ \\
\hline \multicolumn{5}{c}{$\mathbf{1 9 9 9}$ observation } \\
\hline A & $08^{\mathrm{h}} 21^{\mathrm{m}} 46.339^{\mathrm{s}}$ & $-43^{\circ} 02^{\prime} 03.73^{\prime \prime}$ & $0.16^{\prime \prime}$ & $(2.51 \pm 0.38) \times 10^{-3}$ \\
B & $08^{\mathrm{h}} 22^{\mathrm{m}} 23.924^{\mathrm{s}}$ & $-42^{\circ} 57^{\prime} 59.29^{\prime \prime}$ & $0.20^{\prime \prime}$ & $(8.42 \pm 0.75) \times 10^{-3}$ \\
\hline \multicolumn{5}{c}{$\mathbf{2 0 0 5}$ observation $^{\prime}$} \\
\hline A & $08^{\mathrm{h}} 21^{\mathrm{m}} 46.313^{\mathrm{s}}$ & $-43^{\circ} 02^{\prime} 03.46^{\prime \prime}$ & $0.07^{\prime \prime}$ & $(3.20 \pm 0.30) \times 10^{-3}$ \\
B & $08^{\mathrm{h}} 22^{\mathrm{m}} 23.966^{\mathrm{s}}$ & $-42^{\circ} 57^{\prime} 59.70^{\prime \prime}$ & $0.18^{\prime \prime}$ & $(7.47 \pm 0.48) \times 10^{-3}$ \\
\hline
\end{tabular}

${ }^{a}$ The positional errors of sources A and B are given by the PSF fitting and the wavelet detection algorithm respectively.

\section{Observation and data analysis}

Owing to the fact that there are only few bad pixels in the Chandra HRC-I and the pixel size of 0.13187 arcsec oversamples the point spread function (PSF) by a factor of $\sim 5$, the HRC-I appears to be the most suitable detector to perform astrometric measurements of X-ray sources.

Checking the Chandra archive for suitable data we found that by mid of 2006 RX J0822-4300 was observed twice with the HRC-I. The first observation was performed in 1999 December 21 (MJD 51533) for an exposure time of about $16 \mathrm{ks}$. The second observation was done in 2005 April 25 (MJD 53485) for an on-source time of $233 \mathrm{ks}$. In the April 2005 observation, the target was displaced only $\sim 0.2$ arcmin off from the optical axis of the X-ray telescope. In the December 1999 data, it appears to be $\sim 0.3$ arcmin off-axis which, in both cases, is small enough to have a negligible effect for the distortion of the PSF relative to an on-axis observation.

In order to increase the precision required for accurate astrometric measurements, systematic uncertainties need to be corrected. Apart from the aspect offset correction we also considered the errors introduced by determining the event positions. The later included corrections of the tap-ringing distortion in the position reconstruction and the correction of errors introduced by determining the centroid of the charge cloud. Instead of using the fully processed pipeline products, we started our data reduction with level-1 files to be able to correct for these systematic effects. All the data processings were performed with CIAO 3.2.1. Details of the applied corrections are described in the following.

Instabilities in the HRC electronics can lead to a tap-ringing distortion in the position reconstruction of events. Correction has been applied to minimize this distortions in standard HRC level-1 processing which required to know the values of the amplitude scale factor (AMP_SF). Such values are found in the HRC telemetry and are different for each event. Unfortunately, they are often telemetered incorrectly. In order to fix this anomaly, we followed a thread in CIAO to deduce the correct values of AMP_SF in the level-1 event file from other HRC event data and applied these corrected values to minimize the distortion.

The de-gap correction was applied to the event files in order to compensate the systematic errors introduced in the event positions by the algorithm which determines the centroid of the charge cloud exiting the HRC rear micro-channel plate.

After correcting these systematic errors we generated the level-2 event lists files which were used thoroughly for the remaining analysis. We created HRC-I images of RX J0822-4300 for both epochs with a binning factor of 1 so that each pixel has a side length of 0.13187 arcsec.

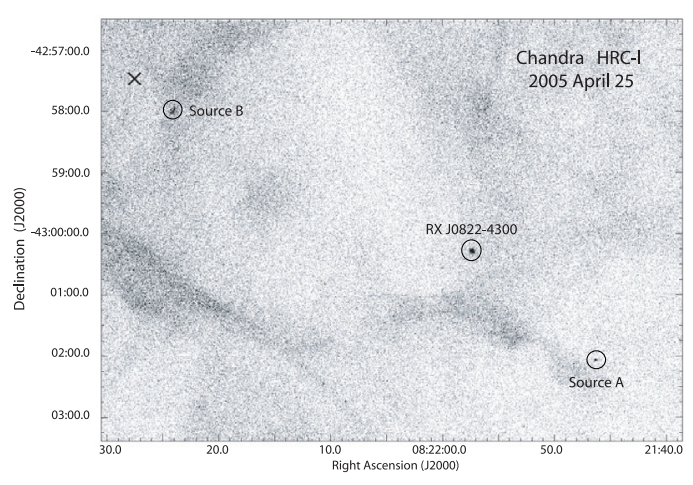

Fig. 1. Part of the Chandra HRC-I image of the Puppis-A supernova remnant. Besides RX J0822-4300, there are two serendipitous point sources detected in the field of view. They are denoted as source A and B. The optical expansion center of Puppis-A as obtained by Winkler et al. (1988) is marked by a cross.

To be able to correct for pointing uncertainties by using X-ray counterparts of stars which have their position known with high accuracy, we applied a wavelet source detection algorithm to the HRC-I images. Two X-ray point sources with a count rate of about $1 \%$ and $3 \%$ relative to that of RX J0822-4300 were detected serendipitously at about $\sim 2.5$ arcmin and $\sim 5.5$ arcmin distance from RX J0822-4300. Figure 1 shows a $9.5 \times 7$ arcmin field surrounding RX J0822-4300 as seen with the HRC-I in April 2005. Both serendipitous sources, denoted as A and B, are indicated in this figure. Their X-ray properties are summarized in Table 1.

In order to determine their X-ray positions with higher accuracy than possible with the wavelet analysis, we fitted a 2D Gaussian model with the modeled PSF as a convolution kernel to both sources $\mathrm{A}$ and $\mathrm{B}$. These fits require some information on the source energy spectrum which is not available from the HRC-I data. We therefore checked the archival XMM-Newton data for both sources and found from an spectroimaging analysis of MOS1/2 data (cf. Fig. 1 in Hui \& Becker 2006) that the hardness-ratios of source A and B are comparable to that of RX J0822-4300 which has its energy peak at $\sim 1.5 \mathrm{keV}$. We therefore extract the Chandra HRC-I PSF model images at $1.5 \mathrm{keV}$ with the corresponding off-axis angles from the CALDB standard library files (F1) by interpolating within the energy and off-axis grids using the CIAO tool MKPSF. The exposure maps were also generated at this energy by using MKEXPMAP.

The size of the $1-\sigma$ error circles of source A obtained by this method are 0.16 arcsec and 0.07 arcsec for the 1999 and 2005 observations, respectively. The relatively large error in the 
December 1999 observation is due to its shorter integration time and thus smaller photon statistics.

The large off-axis angle ( $\sim 5.5 \mathrm{arcmin})$ of source B causes a marked blurring of the PSF (90\% encircled energy radius $\simeq 4 \mathrm{arcsec}$ ). Such distortion makes the source appear to be very dispersed. Given the patchy and uneven supernova remnant emission this source is surrounded by and the limited photon statistics we did not succeed in obtaining its coordinates more accurate than possible with the wavelet algorithm (which is $\sim 0.2$ arcsec). This leaves source A as the only reference star to perform astrometric correction.

Correlating the source position of source A with the Two Micron All Sky Survey (2MASS) catalog (Skrutskie et al. 2006) identified the star with the source designation 08214628-4302037 as a possible optical counterpart. Since the next nearest optical source is located about 5 arcsec away from the X-ray position of source A, we adopt the 2MASS source $08214628-4302037$ as its optical counterpart. The visual magnitudes of the object are $J=12.161 \pm 0.027$, $H=11.675 \pm 0.023$ and $K=11.558 \pm 0.024$. Since its spectral type is not known with certainty, we adopted a typical X-ray-to-optical flux ratio of $\log \left(F_{\mathrm{x}} / F_{\text {opt }}\right) \simeq-2.46$ for stars from Krautter et al. (1999). Assuming a Raymond-Smith thermal plasma model with $k T=0.15 \mathrm{keV}, n_{\mathrm{H}}=4 \times 10^{21} \mathrm{~cm}^{-2}$ (Hui $\&$ Becker 2006) and solar abundances for the star's spectrum we estimated with the aid of PIMMS (version 3.8a2) its HRC-I count rate to be $\sim 3 \times 10^{-3} \mathrm{cts} / \mathrm{s}$. This is in good agreement with the observed count rate of source A (cf. Table 1).

In order to use the optical identification of the serendipitous X-ray source A as a reference source for the offset correction, we have to check whether itself shows a proper motion. To investigate this, we correlated its 2MASS position with the UCAC2 catalog (Zacharias et al. 2003). We unambiguously found a source with the UCAC2 designation 13302738 as a counterpart of the X-ray source A. According to this catalog, this source has a proper motion of $\mu_{\mathrm{RA}}=-16.0 \pm 5.2 \mathrm{mas} / \mathrm{yr}$, $\mu_{\text {dec }}=-1.7 \pm 5.2 \mathrm{mas} / \mathrm{yr}$.

We attempted to make an independent estimate by analysing the images from the first and the second Digitised Sky Surveys ${ }^{1}$. From the observation dates specified for the DSS-1 and DSS-2 images, we found that the epoches of these two images are separated by 5134 days. We took four bright stars within 1 arcmin neighbourhood of the X-ray source A as the references to align the frames of DSS- 1 and DSS-2. None of these stars appeared to be saturated so that their positions could be properly determined by a 2D Gaussian fit. We determined the offset between these two images from comparing the best-fit positions of the four reference stars in both frames. However, we found that the alignment error is at a level of $\sim 0.5$ arcsec. This is close to the average positional discrepancy between DSS-1 and DSS-2 which is found to be $\sim 0.6$ arcsec. The information provided by DSS- $1 / 2$ thus does not allow us to estimate the proper motion of our source of interest independently. In view of this, we can only resort on the findings in the UCAC2 catalog.

Under the assumption that the UCAC2 object 13302738 is indeed the optical counterpart of source A, we applied the aspect correction to the corresponding frames with the proper motion of the reference star taken into account. However, with only one comparison source avaliable for the frame alignment, there are some limitations in our adopted method. Firstly, the roll angle between two frames cannot be determined independently with just one reference source. Hence, the accuracy of the

\footnotetext{
${ }^{1}$ http://ledas-www.star.le.ac.uk/DSSimage/
}

current result is limited by the output of the star-tracking camera, the Pointing Control and Determination system (PCAD). Also, an independent estimate of the HRC-I plate scale cannot be made with only one reference source. The potential variation of the plate scale might introduce an extra error, though we consider this is negligible as the typical uncertainty of the HRC-I plate scale is at the order of $\sim 0.05 \mathrm{mas} / \mathrm{pixel}^{2}$.

The error circle of the UCAC2 object 13302738 is specified to be 0.015 arcsec. Including the uncertainty in the proper motion, the overall positional error of this astrometric source is increased with time which gives 0.016 arcsec and 0.037 arcsec in the December 1999 and April 2005 epoch respectively. The total error of the aspect corrections is calculated by combining the statistical error of the X-ray position of source A and the astrometric error of the UCAC2 object in quadrature. This yields a $1-\sigma$ error of 0.161 arcsec and 0.079 arcsec for the aspect correction of the December 1999 and April 2005 observation, respectively.

The position of RX J0822-4300 was determined by the same procedure we applied to obtain the position for source A. The fits provide us with the coordinates for RX J0822-4300 which are RA $=08^{\mathrm{h}} 21^{\mathrm{m}} 57.389^{\mathrm{s}}$ and Dec $=-43^{\circ} 00^{\prime} 16.90^{\prime \prime}$ (J2000) in the 1999 observation and RA $=08^{\mathrm{h}} 21^{\mathrm{m}} 57.343^{\mathrm{s}}$ and Dec $=-43^{\circ} 00^{\prime} 17.18^{\prime \prime}(\mathrm{J} 2000)$ in the April 2005 observation $^{3}$. The $1-\sigma$ error of the position introduced by the PSF-fit is 0.01 arcsec in both epochs. In order to exclude any dependence of the deduced source positions on the aperture size of the selected source region, we repeated the fits for three different apertures with radii equal to 3 arcsec, 4 arcsec and 5 arcsec, respectively. We did not find any variation of the best-fitted parameters in these independent fittings.

\section{Results and discussion}

The fitted X-ray positions of RX J0822-4300 are plotted for the observational epochs December 1999 and April 2005 in Fig. 2. The size of the error circle for each position is determined by adding the error in correcting the aspect offset and the error of the position of RX J0822-4300 in quadrature. This gives 0.162 arcsec and 0.088 arcsec for the December 1999 and the April 2005 observations, respectively. The corresponding error circles are indicated in Fig. 2.

Comparing the positions of RX J0822-4300 as deduced for the two observations in 1999 and 2005 we found that they are different by $0.574 \pm 0.184$ arcsec. This is well consistent with the displacement estimated from the kinematic age of the SNR and the positional offset of RX J0822-4300 from the SNR's optical expansion center (cf. Sect. 1). The quoted error is $1-\sigma$ and combines the positional errors in both epochs in quadrature.

Given the epoch separation of 5.34 years for both Chandra HRC-I observations the observed proper motion of RX J0822-4300 is $\mu=107.49 \pm 34.46$ mas/yr. The position angle is $\mathrm{PA}=241^{\circ} \pm 24^{\circ}$. Taking the position inferred from the December 1999 observation and the position of the optical expansion center of Puppis-A (Winkler et al. 1988), a position angle of $\mathrm{PA} \simeq 243^{\circ}$ is implied. Such consistency suggests that RX J0822-4300 is indeed physically associated with

\footnotetext{
${ }^{2}$ http://cxc.harvard.edu/cal/Hrma/optaxis/platescale/ geom_public.html

3 The best-fitting position in the 1999 observation has a 0.242 arcsec deviation from that inferred by Hui \& Becker (2006) which corrected the offset by simply using the calculator provided by the Chandra Aspect team http://cxc.harvard.edu/cal/ASPECT/ fix_offset/fix_offset.cgi
} 


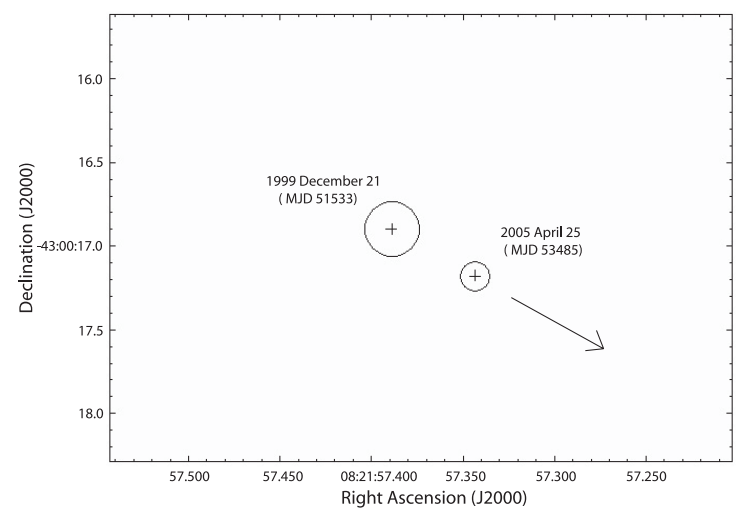

Fig. 2. The best-fitted X-ray positions of RX J0822-4300 in two epochs are marked by crosses. The circles indicate the $1-\sigma$ error. The arrow shows the direction of proper motion inferred from both positions.

Puppis-A and its actual birth place is not too far away from the SNR's optical expansion center. Assuming that the distance of $2.2 \mathrm{kpc}$ to Puppis-A is correct, the observed proper motion implies that RX J0822-4300 has a projected recoil velocity as high as $1121.79 \pm 359.60 \mathrm{~km} \mathrm{~s}^{-1}$ to the southwest. The inferred transverse velocity seems high compared with the average velocity, $\sim 250 \mathrm{~km} \mathrm{~s}^{-1}$, of ordinary radio pulsars (Hobbs et al. 2005) though the large error prevents any constraining conclusions. We would like to mention, though, that there are several fast moving pulsars including PSRs B2011+38 and B2224+64 which have 2D speeds of $\sim 1600 \mathrm{~km} \mathrm{~s}^{-1}$ (Hobbs et al. 2005).

The interesting association of Puppis-A and RX J08224300 provides a unique opportunity to observe the interaction between a compact stellar remnant and a SNR. Also, this system is a test-bed for studying the dynamics of the supernova explosion. Measuring the proper motion of RX J0822-4300 with higher accuracy than possible with the current data will make it possible to provide constrains for the SN explosion model which formed Puppis-A.

Although our result is a promising indication of a fast moving CCO in a SNR, we note that the deduced positional shift is only significant at a $3.1 \sigma$ level. Since the X-ray source A is rather faint, its relatively large positional error predominates in the error budget.
In this letter we have presented the first attempt to measure the proper motion of RX J0822-4300. In order to further constrain this measurement, one or more Chandra HRC-I observations can attain a much larger positional separation in a few years from today. Furthermore, a more accurate proper motion measurement for the optical counterpart of the reference source is also necessary.

After this letter was accepted for publication, we became aware that a paper on the similar subject as presented here was submitted to ApJ by Winkler \& Petre (2006). In their paper, though using the same dataset as we use, a much higher significance $(9.4 \sigma$ !) for the proper motion of RX J0822-4300 is claimed. They justify this by their inclusion of source B as a second reference star. In view of its large off-axis angle, thus blurred PSF and large error circle $(\sim 0.2$ arcsec $)$, we question their justification and doubt their error estimate being a realistic value, even their proper motion estimate is qualitatively similar with ours.

\section{References}

Aschenbach, B. 1998, Nature, 396, 141

Gotthelf, E. V., Halpern, J. P., \& Seward, F. D. 2005, ApJ, 627, 390

Helfand, D. J., \& Becker, R. H. 1984, Nature, 307, 215

Hobbs, G., Lorimer, D. R., Lyne, A. G., \& Kramer, M. 2005, MNRAS, 360, 974

Hui, C. Y., \& Becker, W. 2006, A\&A, 454, 543

Kaspi, V. M., Manchester, R. N., Johnston, S., Lyne, A. G., \& Amico, N. D. 1996, AJ, 111, 2028

Krautter, J., Zickgraf, F.-J., Appenzeller, I., et al. 1999, A\&A, 350, 743

Petre, R., Canizares, C. R., Kriss, G. A., \& Winkler, P. F., Jr. 1982, ApJ, 258, 22 Petre, R., Becker, C. M., \& Winkler, P. F. 1996, ApJ, 465, L43

Pfeffermann, E., \& Aschenbach, B. 1996, in Roentgenstrahlung from the Universe, ed. H. H. Zimmermann, J. Trümper, \& H. Yorke (Garching: MPE), MPE Rep., 263, 267

Seward, F. D., Slane, P. O., Smith, R. K., \& Sun, M. 2003, ApJ, 584, 414

Skrutskie, M. F., Cutri, R. M., Stiening, R., et al. 2006, AJ, 131, 1163

Tananbaum, H. 1999, IAU Circ., 7246

Tuohy, I. R., \& Garmire, G. P. 1980, ApJ, 239, L107

Winkler, P. F., \& Kirshner, R. P. 1985, ApJ, 299, 981

Winkler, P. F., \& Petre, R. 2006 [arXiv:astro-ph/0608205]

Winkler, P. F., Tuttle, J. H., Kirshner, R. P., \& Irwin, M. J. 1988, in Supernova Remnants and the Interstellar Medium, ed. R. S. Roger, \& T. Landecker (Cambridge: Cambridge Univ. Press), 65

Zacharias, N., Urban, S. E., Zacharias, M. I., et al. 2003, VizieR Online Data Catalog, 1289 